\title{
Pressure estimation from single-snapshot tomographic PIV in a turbulent boundary layer
}

\author{
Jan F. G. Schneiders ${ }^{1}$ D $\cdot$ Stefan Pröbsting ${ }^{1} \cdot$ Richard P. Dwight $^{1}$ • \\ Bas W. van Oudheusden ${ }^{1} \cdot$ Fulvio Scarano ${ }^{1}$
}

Received: 25 January 2015 / Revised: 21 January 2016 / Accepted: 22 January 2016 / Published online: 18 March 2016

(C) The Author(s) 2016. This article is published with open access at Springerlink.com

\begin{abstract}
A method is proposed to determine the instantaneous pressure field from a single tomographic PIV velocity snapshot and is applied to a flat-plate turbulent boundary layer. The main concept behind the single-snapshot pressure evaluation method is to approximate the flow acceleration using the vorticity transport equation. The vorticity field calculated from the measured instantaneous velocity is advanced over a single integration time step using the vortex-in-cell (VIC) technique to update the vorticity field, after which the temporal derivative and material derivative of velocity are evaluated. The pressure in the measurement volume is subsequently evaluated by solving a Poisson equation. The procedure is validated considering data from a turbulent boundary layer experiment, obtained with timeresolved tomographic PIV at $10 \mathrm{kHz}$, where an independent surface pressure fluctuation measurement is made by a microphone. The cross-correlation coefficient of the surface pressure fluctuations calculated by the single-snapshot pressure method with respect to the microphone measurements is calculated and compared to that obtained using time-resolved pressure-from-PIV, which is regarded as benchmark. The single-snapshot procedure returns a crosscorrelation comparable to the best result obtained by timeresolved PIV, which uses a nine-point time kernel. When the kernel of the time-resolved approach is reduced to three measurements, the single-snapshot method yields approximately $30 \%$ higher correlation. Use of the method should be cautioned when the contributions to fluctuating pressure from outside the measurement volume are significant. The
\end{abstract}

Jan F. G. Schneiders

j.f.g.schneiders@tudelft.nl

1 Department of Aerospace Engineering, TU Delft, Delft, The Netherlands study illustrates the potential for simplifying the hardware configurations (e.g. high-speed PIV or dual PIV) required to determine instantaneous pressure from tomographic PIV.

\section{Introduction}

In only a decade, techniques that determine the fluid flow pressure based on PIV measurements have come to a degree of maturity that justifies their application in practical problems. These developments have been surveyed in a recent review article by van Oudheusden (2013). Starting from the work of Liu and Katz (2006), who used a dualPIV system to measure velocity and its material derivative and subsequently applied the momentum equation for pressure evaluation, all following studies dealing with instantaneous pressure from PIV have made use of either timeresolved measurements or followed the dual-PIV approach, in order to experimentally determine the velocity material derivative. It has been shown that an accurate determination of the velocity material derivative in turbulent flows requires full three-dimensional evaluation of the velocity and acceleration field, which is currently possible by high-speed tomographic PIV experiments (Ghaemi et al. 2012). Due to uncorrelated random errors in consecutive PIV snapshots, recent studies have employed a Lagrangian pseudo-tracking approach to obtain the velocity material derivative from a series of consecutive time-resolved velocity measurements. For example, Liu and Katz (2013) employed five consecutive velocity fields and Novara and Scarano (2013) applied a PTV technique to eleven consecutive camera images. Other studies have focused on noise reduction of the PIV velocity measurements using for example a POD-based filtering approach (Charonko et al. 
2010) to increase accuracy of the pressure determined from the time-resolved PIV measurements.

The high sampling rate required for time-resolved experiments in airflows compromises the achievable measurement volume of tomographic PIV. According to a recent survey (Scarano 2013), measurement rates achieved in tomo-PIV experiments range from $2.7 \mathrm{kHz}$ (airfoil trailing edge by Ghaemi and Scarano 2011; bluff body wake by de Kat and van Oudheusden 2012) to $5 \mathrm{kHz}$ (turbulent boundary layer by Schröder et al. 2008; rod-airfoil interaction by Violato et al. 2011). More recent experiments in turbulent boundary layers have been conducted at a rate up to $10 \mathrm{kHz}$ (Ghaemi et al. 2012; Pröbsting et al. 2013), at the expense of a further reduction of the measurement volume. The above experiments were conducted with airflow velocities in the range from 7 to $14 \mathrm{~m} / \mathrm{s}$, which poses further restrictions on the value of the Reynolds number. To date, time-resolved tomographic PIV experiments at flow velocities on the order of $100 \mathrm{~m} / \mathrm{s}$ are to be deemed unrealistic, considering that they would require acquisition rates on the order of $100 \mathrm{kHz}$.

The extension of dual-plane PIV (Kähler and Kompenhans 2000) or dual-time PIV (Perret et al. 2006) to dualtomographic PIV systems overcomes the trade-off between measurement volume and recording rate affecting the timeresolved approach, in that it makes use of two low repetition rate lasers and CCD cameras. Such systems also allow investigating flows at higher velocity as one can arbitrarily reduce the time separation between the two velocity measurements, without the restriction set by the repetition rate of a single PIV system. A four-pulse tomographic PIV system has been described (Lynch and Scarano 2014) that can perform acceleration measurements in the compressible flow regime. The drawback is the complexity associated with 8-12 cameras that need to record images from a volume illuminated with two separate dual-pulse lasers.

Alternatives to the time-resolved or dual-PIV approaches are provided in the field of data assimilation. In periodic flows, phase-locked experiments using nontime-resolved PIV systems have been employed in order to obtain pressure from a phase-resolved description of the flow, as reviewed in van Oudheusden (2013). In addition, not relying on phase-locked experiments, Bai et al. (2015) have employed a reduced-order modelling approach using compressive sensing for reconstruction of velocity time-series from PIV measurements performed at limited temporal resolution. More advanced methods reconstruct both velocity and pressure by making use of simulation of the flow governing equations. Suzuki (2012) proposed a reduced-order Kalman filter technique combining PTV and DNS, and later a POD-based hybrid simulation (Suzuki, 2014). Recently, Gronskis et al. (2013), Lemke and Sesterhenn (2013) and Vlasenko et al. (2015) have proposed variational techniques for combination of numerical flow simulations with PIV measurement results. An advantage of these approaches is that they can naturally incorporate also local information from other measurement devices (e.g., surface pressure measurements) as alternative or in addition to PIV. Computational cost associated with the variational or Kalman-filter-based techniques has, however, limited practical application to real-world volumetric experiments.

Less computationally expensive data assimilation approaches solve directly the flow governing equations using the PIV data as initial or boundary conditions. For example, the use of CFD simulations to fill gaps in the measurement domain has been discussed by Sciacchitano et al. (2012). To alleviate measurement rate requirements which limit current pressure-from-PIV setups, Scarano and Moore (2011) proposed to leverage directly the spatial information available by the measurement to increase temporal resolution ("time-supersampling") using a linearized model. The work is based on the assumption of frozen turbulence and advects spatial velocity fluctuations to produce intermediate velocity estimates in between the measured samples. The relatively low computational cost of the linearized model has allowed for demonstration of the method on real-world tomographic PIV data. A similar approach was later used by de Kat and Ganapathisubramani (2013), who discussed the importance of estimating the local convection velocity. To avoid this, the time-supersampling concept was generalized to broader flow regimes (separated flows, vortex-dominated regimes) by Schneiders et al. (2014), who introduced the use of the vortex-in-cell (VIC) technique for time-supersampling of tomographic PIV measurements in incompressible flows. The measured samples of the velocity field are used both as initial and final conditions for a numerical simulation of the vorticity transport equation, which is solved within the measurement domain. The study returned an accurate time-reconstruction, demonstrating that the sampling rate requirements could be significantly reduced with such a procedure.

The objective of the present work moves the attention to the use of VIC on a single velocity field snapshot to estimate the instantaneous pressure field in flows where the pressure fluctuations are dominated by vortical structures in the flow. In particular, the problem of the flat-plate boundary layer is considered, which has been studied in recent studies employing time-resolved tomographic PIV for pressure determination (Ghaemi and Scarano 2011, 2013; Schröder et al. 2011; Pröbsting et al. 2013, amongst others). These studies follow two decades of literature on turbulent boundary layer flows as reviewed in Marusic et al. (2010). Because of the inherent unsteady nature of the turbulent flow structures in the boundary layer, pressure evaluation from tomographic PIV in such flows has only 
been demonstrated using a time-resolved (repetition rate $\sim 10 \mathrm{kHz}$ ) measurement setup.

The single-snapshot pressure evaluation using VIC follows a time-marching approach, whereby a single time step starting from the instantaneous tomographic PIV velocity measurements is needed to approximate the velocity material derivative and subsequently, the instantaneous pressure. As a result, a significant simplification of the measurement systems is potentially obtained, with respect to dual systems for the evaluation of pressure-from-PIV.

\section{Pressure evaluation from a single PIV velocity field}

The discussion here is limited to incompressible and isothermal flows. It is furthermore assumed that the velocity field is measured by tomographic PIV, but the application to data from other $3 \mathrm{D}$ velocity measurement methods is also considered possible. Consider a velocity measurement $\boldsymbol{u}_{m}\left(\boldsymbol{x}, t_{0}\right)$ at time $t=t_{0}$ on a three-dimensional grid $\boldsymbol{x}$ with constant spacing $h$ in the volume $\Omega$, which is assumed a cuboid with boundary $\partial \Omega$. The established time-resolved pressure-from-PIV procedure solves the Poisson equation for pressure (van Oudheusden 2013),

$\nabla^{2} p=-\rho \nabla \cdot \frac{D \boldsymbol{u}}{D t},\left.\quad p\right|_{\partial \Omega_{1}}=f \quad$ and $\left.\quad \frac{\partial p}{\partial n}\right|_{\partial \Omega_{2}}=g$

by approximating $\mathrm{D} u / \mathrm{D} t$ from time-resolved tomographic PIV measurements and with mixed boundary conditions $f$ and $g$ as detailed in Sect. 2.3.1. The present study also employs (1) for pressure evaluation, but approximates $\mathrm{D} u / \mathrm{D} t$ from a single snapshot. It should be remarked that for detailed assessment of alternative, established pressurefrom-time-resolved-PIV techniques, the reader is referred to Charonko et al. (2010) and van Oudheusden (2013). In the present study, second-order central differences are used in the interior domain and first-order single-sided differences on the domain boundaries. The discretized gradient operator is denoted by $\nabla_{h}$ and the discretized Laplacian by $\nabla_{h}^{2}$. The subscript $h$ is used to denote the discretized fields and operators. The computational grid is Cartesian and chosen equal to the PIV measurement grid. The discretized Poisson equation for pressure becomes,

$\nabla_{h}^{2} p_{h}=-\left.\rho \nabla_{h} \cdot \frac{D \boldsymbol{u}}{D t}\right|_{h},\left.\quad p_{h}\right|_{\partial \Omega_{1}}=f_{p}$ and $\left.\quad \frac{\partial p}{\partial n}\right|_{h, \partial \Omega_{2}}=g_{p}$

The Poisson equation for pressure is discretized using second-order central differences. Ghost points at the external side of the domain boundary are eliminated through the Neumann boundary condition (see e.g. Ebbers and Farnebäck 2009).

For the unsteady flow regime, time-resolved measurements are typically performed to provide ${ }^{\mathrm{D} u} /_{\mathrm{D} t \mathrm{th}}$ in the conventional pressure determination approach. Here, it is proposed to approximate the velocity material derivative from a single tomographic PIV snapshot by a vortex-in-cell (VIC) simulation (Sect. 2.1).

\subsection{Approximation of $\mathrm{D} u / \mathrm{D} t$ from single velocity snapshot}

From a tomographic PIV velocity measurement $\boldsymbol{u}_{m}\left(\boldsymbol{x}, t_{0}\right)$, vorticity is approximated on the measurement grid,

$\boldsymbol{\omega}_{h}=\nabla_{h} \times \boldsymbol{u}_{m}$.

Following the VIC procedure outlined in Schneiders et al. (2014), the divergence-free approximation of the measured velocity field is calculated by solution of,

$\nabla_{h}^{2} \boldsymbol{u}_{h}=-\nabla_{h} \times \omega_{h},\left.\quad \boldsymbol{u}_{h}\right|_{\partial \Omega}=\boldsymbol{u}_{m}$.

Although imposing the divergence-free condition has been demonstrated as an effective tool for noise reduction of 3D data (e.g. by de Silva et al. 2013; Schiavazzi et al. 2014), in the present study, the divergence-free approximation is an inherent step of the procedure and is not meant for preconditioning or noise reduction of the measured velocity field. In the interior domain, typically $\boldsymbol{u}_{h} \neq \boldsymbol{u}_{m}$, which is mostly ascribed to measurement errors. Recent studies have proposed to estimate the measurement error with the difference between a divergence-free flow field and $\boldsymbol{u}_{m}$ (Atkinson et al. 2011; Lynch and Scarano 2014; Sciacchitano and Lynch 2015, amongst others).

The temporal derivative of vorticity can subsequently be calculated by a finite-difference discretization of the inviscid vorticity transport equation,

$\left.\frac{\partial \omega}{\partial t}\right|_{h, E u l}=\left(\boldsymbol{\omega}_{h} \cdot \nabla_{h}\right) \boldsymbol{u}_{h}-\left(\boldsymbol{u}_{h} \cdot \nabla_{h}\right) \boldsymbol{\omega}_{h}$.

The subscript Eul stands here for Eulerian, as later an alternative discretization (using VIC) will be introduced. Approximation of $\partial \boldsymbol{\omega} / \partial t$ using (5) allows for approximation of the temporal velocity derivative, $\left.\frac{\partial u}{\partial t}\right|_{h}$, by solution of

$\left.\nabla_{h}^{2} \frac{\partial \boldsymbol{u}}{\partial t}\right|_{h}=-\nabla_{h} \times\left.\frac{\partial \boldsymbol{\omega}}{\partial t}\right|_{h},\left.\quad \frac{\partial \boldsymbol{u}}{\partial t}\right|_{h}=f_{d u}$ on $\partial \Omega$,

with Dirichlet boundary conditions $f_{d u}$ as discussed in Sect. 2.3.2. The velocity material derivative is subsequently approximated by,

$\left.\frac{D \boldsymbol{u}}{D t}\right|_{h} ^{\text {single-snapshot }}=\left.\frac{\partial \boldsymbol{u}}{\partial t}\right|_{h}+\left(\boldsymbol{u}_{h} \cdot \nabla_{h}\right) \boldsymbol{u}_{h}$. 
It should be remarked that solution of (5) requires approximation of the gradient of vorticity. VIC avoids this by employing a vortex particle discretization, as discussed in Schneiders et al. (2014). The VIC time-supersampling procedure detailed in the latter paper yields, when applied to an instantaneous measurement, $\boldsymbol{\omega}_{h}\left(\boldsymbol{x}, t_{0}+\Delta t\right)$ directly from a single forward-time integration in the interior domain. Using single-sided finite differences, the temporal vorticity derivative is subsequently approximated,

$\left.\frac{\partial \boldsymbol{\omega}}{\partial t}\right|_{h, V I C}=\frac{\omega_{h}\left(\boldsymbol{x}, t_{0}+\Delta t\right)-\boldsymbol{\omega}_{h}\left(\boldsymbol{x}, t_{0}\right)}{\Delta t}$.

The integration time step is chosen on the order of the PIV pulse time separation; sufficiently small to avoid truncation errors, but large enough to avoid rounding errors. On the two grid points adjacent to each volume boundary, the VIC procedure requires boundary values (as discussed in detail in Schneiders et al. 2014), which are here taken from (5),

$\left.\frac{\partial \omega}{\partial t}\right|_{h}=\left\{\begin{array}{l}\left.\frac{\partial \omega}{\partial t}\right|_{h, V I C} 2<i<L, 2<j<M, \text { and } 2<k<N \\ \left.\frac{\partial \omega}{\partial t}\right|_{h, E u l} \text { otherwise }\end{array}\right.$

where $i \in\{1, \ldots, L\}, j \in\{1, \ldots, M\}$ and $k \in\{1, \ldots, N\}$ are the grid points indices in the computational volume. When (9) is employed instead of (5) in the full domain, this improves the pressure computation, as reflected by the slight increase in correlation coefficient as witnessed in the experimental assessment (Sect. 4).

\subsection{Range of application and limitations}

The first limitation regards reconstruction of pressure in flow cases where the pressure associated with the potential field dominates pressure associated with the vorticity field. Consider the Helmholtz decomposition of velocity into curl free and divergence-free parts,

$\boldsymbol{u}^{p}=-\nabla \Phi$

$\boldsymbol{u}^{v}=\nabla \times \boldsymbol{A}$

$\boldsymbol{u}=\boldsymbol{u}^{p}+\boldsymbol{u}^{v}$

With this decomposition, Eq. (4) can be rewritten into

$$
\begin{aligned}
\nabla^{2}\left(u^{p}+u^{v}\right) & =-\nabla \times \omega,\left.\quad\left(u^{p}+u^{v}\right)\right|_{\partial \Omega}=u_{m} \\
& =-\nabla \times\left(\nabla \times u^{p}\right)_{=0}-\nabla \times\left(\nabla \times u^{v}\right)
\end{aligned}
$$

Thus, the source term of the Poisson equation to reconstruct velocity only contains $\boldsymbol{u}^{v}$ and therefore the potential field $\boldsymbol{u}^{p}$ is reconstructed using information from the boundary points only. As there is typically a much smaller number of points on the boundary than in the interior, measurement errors on the domain boundaries are expected to affect $\boldsymbol{u}^{p}$ more significantly than $\boldsymbol{u}^{v}$. In flow cases where $\boldsymbol{u}^{p}$ dominates, sensitivity of the reconstructed potential field to the domain boundary conditions should be cautioned. The present manuscript, however, focusses on a flow case dominated by $\boldsymbol{u}^{v}$.

The second limitation follows from the fact that the proposed technique can only use the information available from a single velocity measurement to approximate the velocity temporal derivative and subsequently pressure. The limitations of the technique become apparent upon splitting of Eq. (6) into a non-homogeneous Poisson equation with homogeneous boundary conditions and a homogeneous Poisson equation with non-homogeneous boundary conditions,

$\left.\nabla^{2} \frac{\partial \boldsymbol{u}}{\partial t}\right|_{A}=-\nabla_{h} \times \frac{\partial \boldsymbol{\omega}}{\partial t},\left.\quad \frac{\partial \boldsymbol{u}}{\partial t}\right|_{A}=0$ on $\partial \Omega$,

$\left.\nabla^{2} \frac{\partial \boldsymbol{u}}{\partial t}\right|_{B}=0,\left.\quad \frac{\partial \boldsymbol{u}}{\partial t}\right|_{B}=f_{d u}$ on $\partial \Omega$

$\frac{\partial \boldsymbol{u}}{\partial t}=\left.\frac{\partial \boldsymbol{u}}{\partial t}\right|_{A}+\left.\frac{\partial \boldsymbol{u}}{\partial t}\right|_{B}$.

Equation (12a) can be solved directly from the temporal vorticity derivative approximated by (5) from a single tomographic PIV velocity snapshot. However, Eq. (12b) cannot be readily solved in the absence of knowledge about the boundary conditions on the temporal velocity derivative, which is not measured by the PIV system. When boundary conditions on (12b) cannot be approximated, the pressure field can only be determined up to the pressure induced by the irrotational acceleration field following from Eq. (12b). To illustrate this, consider the extreme case where pressure is determined solely by this component. Take for example the flow in a cylinder, which is uniformly accelerated by a piston: a uniform pressure gradient is associated with the acceleration caused by the piston motion. As a result, the absolute value of the pressure cannot be determined unless, for this example, the piston path is known, or in general, when the fluid flow acceleration at the domain boundary can be estimated. For many relevant applications in the turbulent flow regime, the instantaneous value of $\partial \boldsymbol{u} / \partial t$ is dominated by the contribution from Eq. (12a). Such cases include turbulent boundary layers, flow over stationary airfoils, wakes and jets. In addition to the above considerations, in Sect. 2.3.2, three types of boundary conditions are proposed to approximate boundary conditions for Eq. (12b) for a wider variety of cases. 


\subsection{Treatment of boundary conditions}

For the present problem, the treatment of boundary conditions (BC) needs to be considered at two stages in the procedure: first for the Poisson equation for pressure (2) and second for the solution of the Poisson equation for the velocity temporal derivative (6).

\subsubsection{Pressure boundary conditions}

Mixed BCs on pressure are generally employed in PIVbased pressure determination methods, with a Dirichlet $\mathrm{BC} f_{p}$ on $\partial \Omega_{1}$ and Neumann BC $g_{p}$ on $\partial \Omega_{2}$. The Dirichlet boundary condition $f_{p}$ may be obtained from additional experimental data, using pressure probes or surface pressure transducers. Alternatively, a more practiced approach is including in the measurement domain regions where the flow is known to be irrotational and possibly steady. In that case, pressure-velocity models as simple as the Bernoulli equation or isentropic relations may be employed (see e.g. Kurtulus et al. 2007; Ragni et al. 2009; de Kat and van Oudheusden 2012). The use of such a model for pressure yields a Dirichlet $\mathrm{BC} f_{p}$ along an extended region $\partial \Omega_{1}$ of one or more volume boundaries.

Neumann boundary conditions $g_{p}$ are provided by the momentum equation, which in discretized form becomes,

$g_{p}=-\rho \frac{\mathrm{D} \boldsymbol{u}}{\mathrm{D} t}+\mu \nabla_{h}^{2} \boldsymbol{u} \approx \nabla p$.

For pressure evaluation, the viscous terms are typically neglected (van Oudheusden, 2013). Ghaemi et al. (2012) have directly evaluated the viscous terms from a PIV measurement in a turbulent boundary layer and showed that in a turbulent boundary layer these terms are typically two orders of magnitude smaller than the other terms in the momentum equation. It may be remarked that the viscous terms are only neglected for the computation of the instantaneous pressure. The measured velocity field inherently incorporates the physical effects of viscosity.

\subsubsection{Velocity acceleration boundary conditions}

Boundary conditions for (6) are not measured by the singlesnapshot PIV experiment or readily provided by the system of equations, in contrast to the Neumann type BC for pressure. When the measurement volume boundary involves a free stream or steady flow, $\partial \boldsymbol{u} / \partial t=0$ can be imposed there. Similarly, at a wall the no-slip BC also implies $\partial \boldsymbol{u} / \partial t=0$. However, where the volume boundaries involve unsteady flow regions, a model for the unsteady boundary conditions is required depending on the flow case under consideration, as discussed in detail in Sect. 2.2. These models approximate the temporal velocity derivative on the domain boundary to account for boundary effects. In the interior domain, the VIC model approximates the temporal velocity derivative by simulation of the vorticity captured in the measurement volume. Three types of approximations for Dirichlet boundary conditions will be considered in the present study; in the experimental assessment, the sensitivity of the solution to the different approximations is assessed.

1. Convection boundary conditions of the form,

$$
\frac{\partial \boldsymbol{u}}{\partial t}=-\left(\boldsymbol{u}_{c} \cdot \nabla\right) \boldsymbol{u},
$$

which are expected to be accurate when the assumption of "frozen turbulence" holds on the boundary region and small velocity fluctuations are convected by a larger mean convection $\boldsymbol{u}_{c}$ velocity (Taylor's hypothesis). The problem of determining the correct value of the convective velocity has been addressed over the past decades (e.g. Wills 1964; Krogstad et al. 1998; de Kat and Ganapathisubramani 2013). For conciseness, however, in the experimental assessment (Sect. 4), the local instantaneous flow velocity is used as an estimate for the convection velocity. It should be remarked that similar models have also been used for boundary conditions for pure numerical simulations (e.g. Orlanski 1976) and the model has recently been employed by Gronskis et al. (2013) in an effort to combine direct numerical simulation with PIV measurements.

2. Padding boundary conditions: when the vorticity outside of the measurement volume is small compared to the vorticity contained within the measurement volume, the measurement volume may be padded with an extension region of zero vorticity and a homogeneous boundary condition on the acceleration is prescribed on the enlarged domain, which allows the temporal velocity derivative on the measurement domain volume to become non-zero. This procedure is illustrated in more detail in Sect. 3 using a numerical example. It should be remarked that this boundary condition type is also used in pure numerical simulations using the vortex-incell technique (e.g. Cottet and Poncet 2003).

3. Homogeneous boundary conditions: when $\partial \boldsymbol{u} / \partial t \approx 0$ on the volume boundaries, the homogeneous boundary condition $\partial \boldsymbol{u} / \partial t=0$ is a trivial approximation. Additionally, this boundary condition is considered in the present investigation to assess sensitivity of the result when a homogeneous boundary condition is prescribed.

These three boundary condition types will all be considered in the experimental assessment (Sect. 4) using independent microphone measurement data to establish the sensitivity of the solution to a change in boundary conditions. 
In the next section, the use of the padding type of boundary condition will be illustrated for the numerical test case of an advecting Gaussian vortex.

\section{Numerical illustration}

Consider a two-dimensional Gaussian vortex being advected at a constant velocity $u_{c}$ and positioned in the centre of a simulated measurement domain at time $t_{0}$ (Fig. 1a). This case has been used previously for assessment of timeresolved PIV pressure evaluation procedures by amongst others de Kat and van Oudheusden (2012) and Lynch and Scarano (2014). The tangential velocity field induced by the Gaussian vortex blob is given by,

$V_{\theta}=\frac{\Gamma}{2 \pi r}\left(1-e^{-\frac{r^{2}}{c_{\theta}}}\right)$,

where $\Gamma$ is the circulation and $c_{\theta}=r_{c}^{2} / \gamma$. Choosing $\gamma=1.256$, the tangential velocity peaks at the core radius $r_{c}$. A positive uniform velocity $u_{c}$ is added to the velocity field. For this illustrative case, $r_{\mathrm{c}} / L=0.25$ and $u_{\mathrm{c}} L / \Gamma=2$, with $L$ being the width of the square measurement domain. The analytical expression for the exact pressure field centred on the vortex core is given by

$p=-\frac{1}{2} \rho V_{\theta}^{2}-\frac{\rho \Gamma^{2}}{4 \pi^{2} c_{\theta}}\left(E i\left(\frac{r^{2}}{c_{\theta}}\right)-E i\left(\frac{2 r^{2}}{c_{\theta}}\right)\right)$,

with $E i(x)$ the exponential integral function. For reference, the exact $\partial \boldsymbol{u} / \partial t$ and pressure fields are plotted in, respectively, Fig. 2a, e. It should be remarked that the pressure fields given in this section are unique up to a constant and to allow for comparison to the exact pressure they are fixed to zero in the domain centre.

Consider now a simulated single-snapshot measurement of the exact instantaneous velocity field in a measurement domain equal to the region plotted in Fig. 1a $\left(-2<x / r_{\mathrm{c}}<2,-2<y / r_{\mathrm{c}}<2\right)$. When pressure is calculated directly from this velocity field, neglecting the $\partial \boldsymbol{u} / \partial t$ term
Fig. 1 Vorticity field in the simulated measurement domain (a), exact temporal vorticity derivative (b) and the temporal derivative of vorticity calculated by VIC from the single velocity measurement (c)
Fig. 2 Temporal derivative of streamwise velocity (top) and pressure (bottom); a, e exact, $\mathbf{b}, \mathbf{f}$ assuming $\partial \boldsymbol{u} / \partial t=0, \mathbf{c}, \mathbf{g}$ single-snapshot VIC without boundary padding and $\mathbf{d}, \mathbf{h}$ with boundary padding
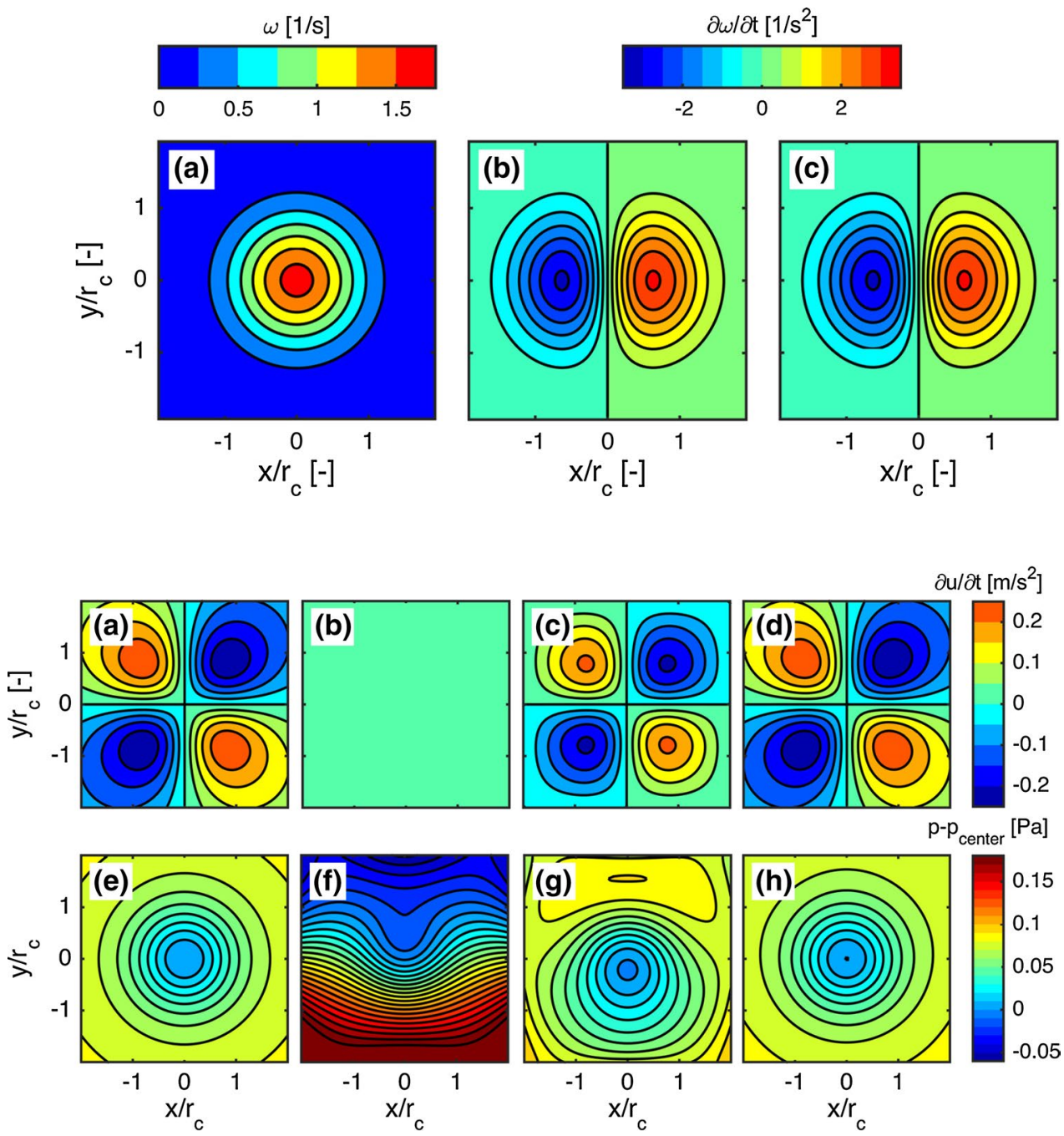
using the steady Poisson equation with Neumann boundary conditions, this leads to an unsatisfactory result as can be seen directly by comparison of Fig. 2e (exact pressure) and Fig. 2f (approximated pressure neglecting $\partial \boldsymbol{u} / \partial t$ ).

The proposed single-snapshot method aims to improve upon this by approximating $\partial \boldsymbol{u} / \partial t$. The temporal vorticity derivative estimated with the VIC method is given in Fig. 1c. The temporal derivative of vorticity is positive to the right of the vortex blob and negative to its left, according to the motion of the vortex blob to the right. The temporal velocity derivative is subsequently calculated by solution of Poisson Eq. (6). Figure 2a shows in this test case that $\partial \boldsymbol{u} / \partial t$ on the measurement domain boundary is nonzero. Prescribing $\partial \boldsymbol{u} / \partial t=0$ on the boundaries for solution of (6) forces, the temporal velocity derivative to zero near the domain boundaries (Fig. 2c). Nevertheless, an improved approximation of the exact pressure field is obtained in comparison with neglecting $\partial \boldsymbol{u} / \partial t$ entirely, as can be seen upon comparison of Fig. 2f, g.

To obtain a further improvement of the approximated pressure field, note that vorticity outside of the simulated measurement domain is practically zero (Fig. 1a). This can be exploited for solution of (6), by using padding type boundary conditions (Sect. 2.3.2). The approximated temporal derivative of vorticity calculated is padded with zeros around the domain, enlarging the computational domain (Fig. 3, left figure). The size of the padded region should account for the length scale of flow fluctuations and is in the present case chosen equal to the size of the vortex in the measurement domain $\left(2 r_{\mathrm{c}}\right)$. Hence, the domain is extended on all sides by $2 r_{\mathrm{c}}$. Prescribing the value of the temporal derivative to zero on the extended computational boundary for solution of (6) allows the temporal derivative to attain nonzero values in the actual measurement domain (Fig. 3, right figure). The results show that a more accurate approximation of the exact temporal derivative can be obtained with this approach (Fig. 2d). Also, the pressure field evaluated from a single velocity snapshot with VIC and boundary padding is reasonably accurate (Fig. $2 \mathrm{~h}$ ) and improves further upon the result without zero-padding (Fig. $2 \mathrm{~g}$ ).

The padding boundary condition and the other two boundary conditions proposed in Sect. 2.3.2 are evaluated in a more realistic case in the next section, where the procedure is applied to a real tomographic PIV experiment in a turbulent boundary layer and validated against independent microphone pressure fluctuation measurements.

\section{Experimental assessment}

The experimental assessment employs the turbulent boundary layer tomographic PIV measurements of Pröbsting et al. (2013), where pressure-from-time-resolved PIV was compared to a surface-mounted pressure transducer. In the above study, the two independent measurements yielded a maximum cross-correlation coefficient of 0.6 . This value repeats that obtained earlier by Ghaemi et al. (2012) under a more favourable measurement configuration. In the present validation of the single-snapshot method, the procedure follows the above studies, using the cross-correlation coefficient as a metric of measurement accuracy.

The experiment considers a turbulent boundary layer on a flat plate (Fig. 4) at a free stream velocity of $10 \mathrm{~m} / \mathrm{s}$, corresponding to a Reynolds number based on the local boundary layer thickness $(\delta=9.4 \mathrm{~mm})$ of $\operatorname{Re}_{\delta}=6240$. The tomographic PIV measurements are performed at $10 \mathrm{kHz}$ with four LaVision HighSpeedStar CMOS cameras equipped with Nikon Micro-Nikkor $105 \mathrm{~mm}$ prime lenses and a Quantronix Darwin Duo Nd:YLF laser. A multi-pass light amplification system is installed, following the indications of Ghaemi and Scarano (2010) to increase the illumination intensity. Knife edges are employed to obtain a top-hat intensity profile and avoid attenuation of laser intensity near volume boundaries. To obtain the vector field, the sequence of objects is analysed with a volume deformation iterative multigrid technique and boundary vectors are cropped to avoid loss-of-correlation
Fig. 3 Domain padding applied to the temporal vorticity derivative computed by VIC (left) and the corresponding $\partial \boldsymbol{u} / \partial t$ computed with homogeneous $\mathrm{BC}$ (right); the measurement domain is given by the dashed red line
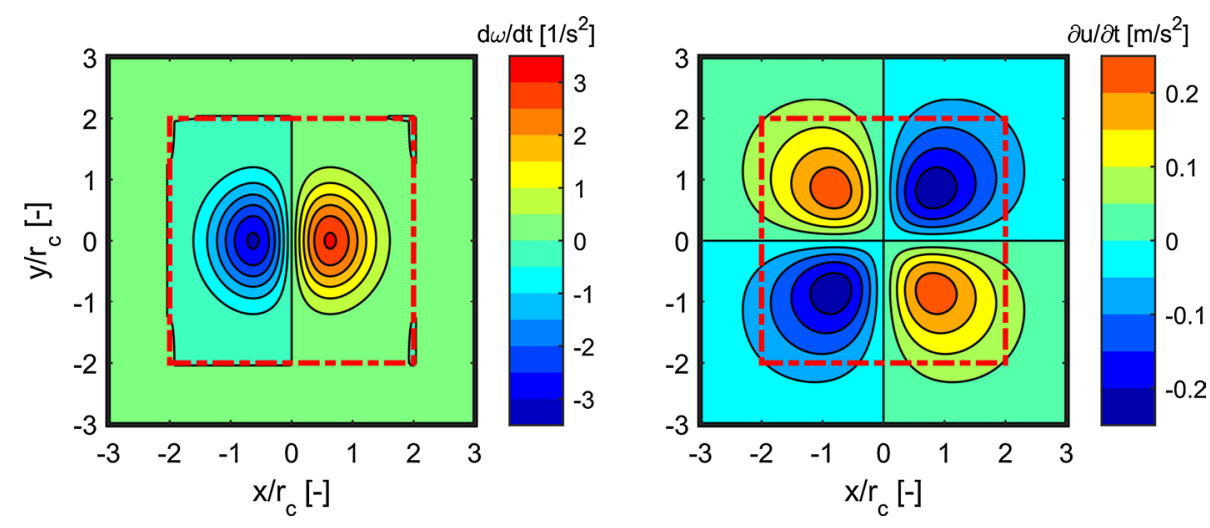


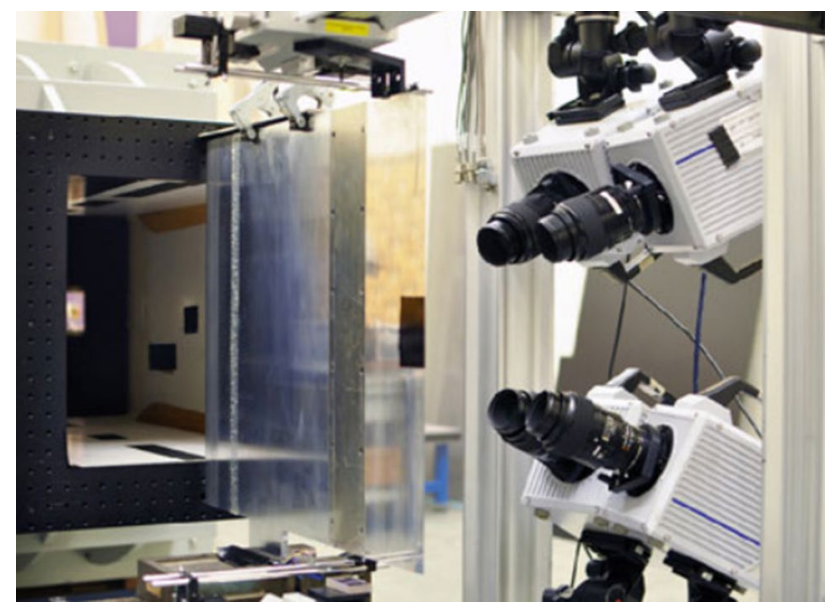

Fig. 4 Photograph of the tomographic PIV experiment (figure reproduced from Pröbsting et al. 2013)

Table 1 Measurement conditions

\begin{tabular}{lll}
\hline Free stream velocity & $\mathrm{V}_{\infty}$ & $10 \mathrm{~m} / \mathrm{s}$ \\
\hline Reynolds number & $R e_{\delta}$ & 6240 \\
& $R e_{\theta}$ & 723 \\
& $R e_{\tau}$ & 436 \\
Shape factor & $H$ & 1.45 \\
Boundary layer thickness & $\delta$ & $9.4 \mathrm{~mm}$ \\
Displacement thickness & $\delta^{*}$ & $1.5 \mathrm{~mm}$ \\
Momentum thickness & $\theta$ & $1.1 \mathrm{~mm}$ \\
Wall shear velocity & $u_{\tau}$ & $0.52 \mathrm{~m} / \mathrm{s}$ \\
\hline
\end{tabular}

Table 2 Tomographic PIV configuration

\begin{tabular}{ll}
\hline Reconstructed volume & $L_{x} \times L_{\mathrm{y}} \times L_{z}=19 \times 4 \times 41 \mathrm{~mm}^{3}$ \\
Magnification & 0.45 \\
Interrogation volume size & $l_{x} \times l_{y} \times l_{z}=32 \times 16 \times 32$ vox \\
Overlap & $75 \%$ \\
Vector spacing & $\Delta x=\Delta z=0.33 \mathrm{~mm}, \Delta y=0.16 \mathrm{~mm}$ \\
Acquisition frequency & $10 \mathrm{kHz}$ \\
Number of samples & 1500 \\
\hline
\end{tabular}

effects. The surface pressure fluctuations are measured simultaneously with the PIV measurements at a single location within the measurement volume using a Sonion 8010T condenser microphone. Salient details of the experiment are given in Tables 1 and 2 and sketches of the setup are given in Fig. 5. For a more complete discussion, the reader is referred to Pröbsting et al. (2013). In the next section, first the benchmark time-resolved pressure evaluation procedure is outlined. Subsequently, in Sect. 4.2, results of the experimental assessment are discussed.

\subsection{Benchmark time-resolved pressure evaluation}

The time-resolved pressure evaluation procedure is chosen equal to the procedure used originally by Pröbsting et al. (2013), allowing for direct comparison of the results. The latter paper employed the following stencil for approximation of the velocity material derivative,

$\left.\frac{D \boldsymbol{u}}{D t}\right|_{\mathrm{TR}-\mathrm{PIV}}=\left(\boldsymbol{\Delta} \boldsymbol{t}^{T} \boldsymbol{\Delta} \boldsymbol{t}\right)^{-1} \boldsymbol{\Delta} \boldsymbol{t}^{T} \boldsymbol{\Delta} \boldsymbol{u}$,

where $\Delta \boldsymbol{t}=\left[\Delta t_{-j}, \ldots, \Delta t_{j}\right]^{T}$, with $\Delta t_{j}=t_{j}-t_{0}$ and similarly for $\boldsymbol{\Delta} \boldsymbol{u}$, with $\Delta \boldsymbol{u}_{i}=\boldsymbol{u}\left(\boldsymbol{x}_{\boldsymbol{p}}\left(t_{i}\right), t_{i}\right)-\boldsymbol{u}\left(\boldsymbol{x}_{\boldsymbol{p}}\left(t_{0}\right), t_{0}\right)$, where,

$\boldsymbol{x}_{\boldsymbol{p}}\left(t_{ \pm j}\right)= \pm \boldsymbol{u}\left(\boldsymbol{x}_{\boldsymbol{p}}\left(t_{ \pm(j-1)}\right), t_{ \pm(j-1)}\right) \Delta t+\boldsymbol{x}_{\boldsymbol{p}}\left(t_{ \pm(j-1)}\right)$,

and $j=1, \ldots, \mathrm{M}$.

Pröbsting et al. (2013) found the $M=4$ nine-snapshot stencil to be optimal for the present experimental dataset. The present manuscript does not aim to improve the timeresolved pressure evaluation procedure, but proposes a pressure evaluation procedure for non-time-resolved PIV measurements and therefore the case of $M=4$ is taken as reference and benchmark result. In addition, a smaller three-snapshot stencil $(M=1)$ will be considered for comparison, which is illustrative for dual-PIV cases where only three to four consecutive measurements are available instead of nine. For a more extensive discussion on timeresolved PIV pressure evaluation methods, the reader is referred to van Oudheusden (2013) and references therein.

It should be remarked that due to the Lagrangian nature of the material derivative evaluation, the procedure does not yield values near the inflow and outflow boundaries as information from outside the measurement domain would be required in these regions. The extent of this region can be approximated by

$L_{c r} \approx \frac{u_{\infty} M}{\Delta x}$,

and the measurement volume is cropped by this region, $12 \Delta x$, on both in- and outflow. In addition, a crop of $5 \Delta x$ is applied on both sides in spanwise direction of $2 \Delta y$ and $5 \Delta y$ on, respectively, the bottom and top surfaces of the volume. The extent of the domain crop is sketched also in Fig. 6.

For pressure evaluation in the cropped volume, mixed boundary conditions are employed for the Poisson equation for pressure. Neumann boundary conditions given by (13) are prescribed on all boundaries, except the top boundary $(y / \delta=0.4)$, where Dirichlet conditions are prescribed based on an extended version of the Bernoulli equation, corrected for an unsteady convective perturbation as proposed by de Kat and van Oudheusden (2012),

$p_{\text {top }}^{\prime}=-\frac{1}{2} \rho\left(\boldsymbol{u}^{\prime} \cdot \boldsymbol{u}^{\prime}\right)=f_{p}$ 
Top View measurement setup

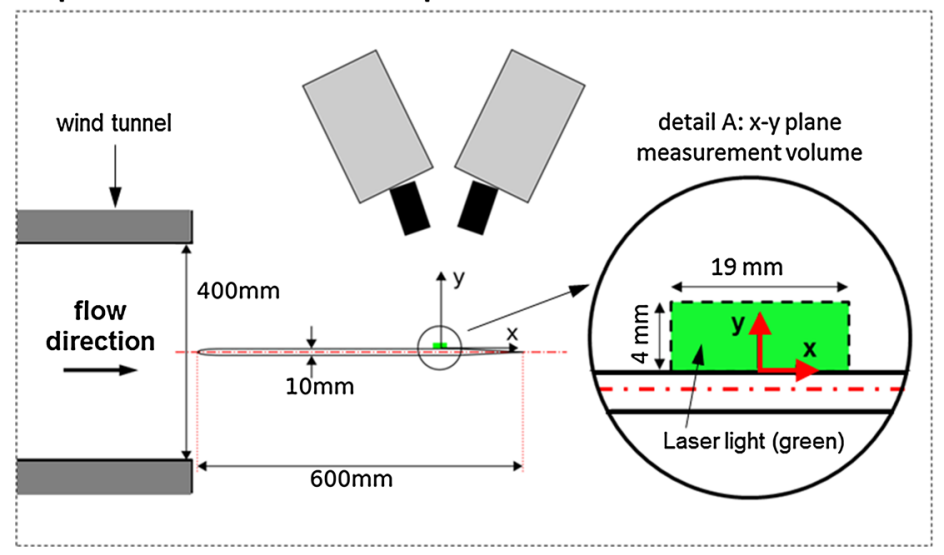

Back View measurement setup

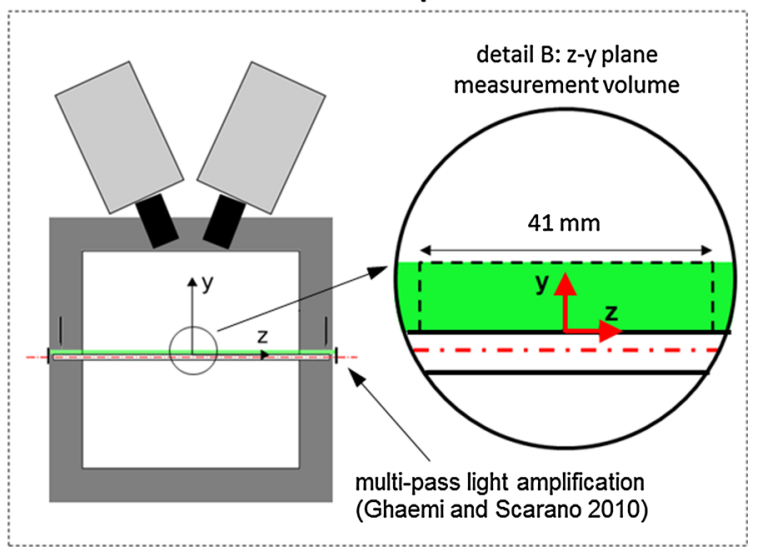

Fig. 5 Schematic of the tomographic PIV experiment (schematics not to scale); top view (left) and back view (right); the measurement volume is indicated by the black dashed line in details A and B
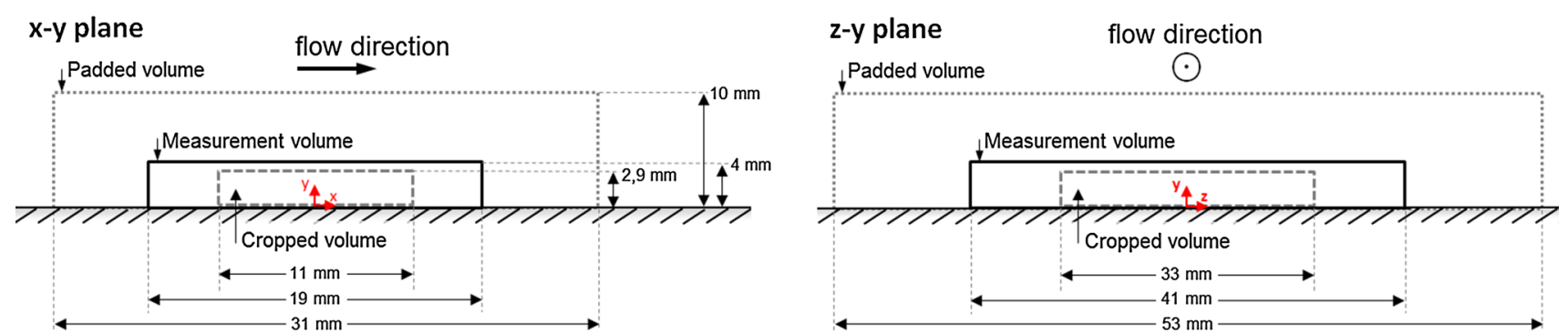

Fig. 6 Overview of the measurement, cropped and padded volumes (schematic not to scale)

\subsection{Results}

First, the pressure-from-time-resolved PIV results are discussed to provide a benchmark for the proposed singlesnapshot method (Sect. 4.2.1), after which the single-snapshot results (Sect. 4.2.2) are discussed.

\subsubsection{Benchmark time-resolved results}

A single instantaneous pressure field in the plane parallel to the wall at $y / \delta=0.2$ evaluated using the time-resolved procedure with the three $(M=1)$ and nine $(M=4)$ snapshot stencil is plotted in, respectively, Fig. 7a, b. It is expected that the result with $M=1$ is strongly affected by random errors in the velocity measurements, which can also be observed in Fig. 4a, b by comparison of the two results. In these figures, the free stream pressure level $\mathrm{p}_{\text {ref }}$ has been subtracted from the fields. For validation, the approximated pressure fluctuations at the microphone location are compared to the simultaneous instantaneous microphone surface pressure measurements (Fig. 8), where both the PIV and microphone results are band-pass-filtered for $300 \mathrm{~Hz}<f<3 \mathrm{kHz}$ (analogous to Pröbsting et al. 2013).
For computation of the cross-correlation coefficients, the microphone signal is sub-sampled after application of the band-pass filter to match the sampling frequency of the time-resolved tomographic PIV measurement.

Comparison to the microphone surface pressure measurement in the centre of the measurement domain (Fig. 8, grey line) confirms low correlation to the reference microphone signal of the time-resolved pressure-from-PIV result using a small three-snapshot stencil $(M=1$, blue line) and the peak value of the correlation coefficient is only $R_{\mathrm{pp}^{\prime}}=0.45$ (Table 3). This result is significantly improved when the larger stencil of $M=4$ is considered. Similarly to the results reported in Pröbsting et al. (2013), in the present study a correlation peak of $R_{\mathrm{pp}^{\prime}, M 4}=0.65$ is found with this stencil.

\subsubsection{Single-snapshot pressure results}

First, the single-snapshot pressure is calculated without the proposed procedure for approximation of $\partial \boldsymbol{u} / \partial t$. The flow regime is incompressible $(\mathrm{Ma}=0.03)$, and the velocity field and $\partial \boldsymbol{u} / \partial t$ are expected to be divergence free. Consequently, $\partial \boldsymbol{u} / \partial t$ drops out of the incompressible Poisson equation for pressure, and hence, it may be argued that it 

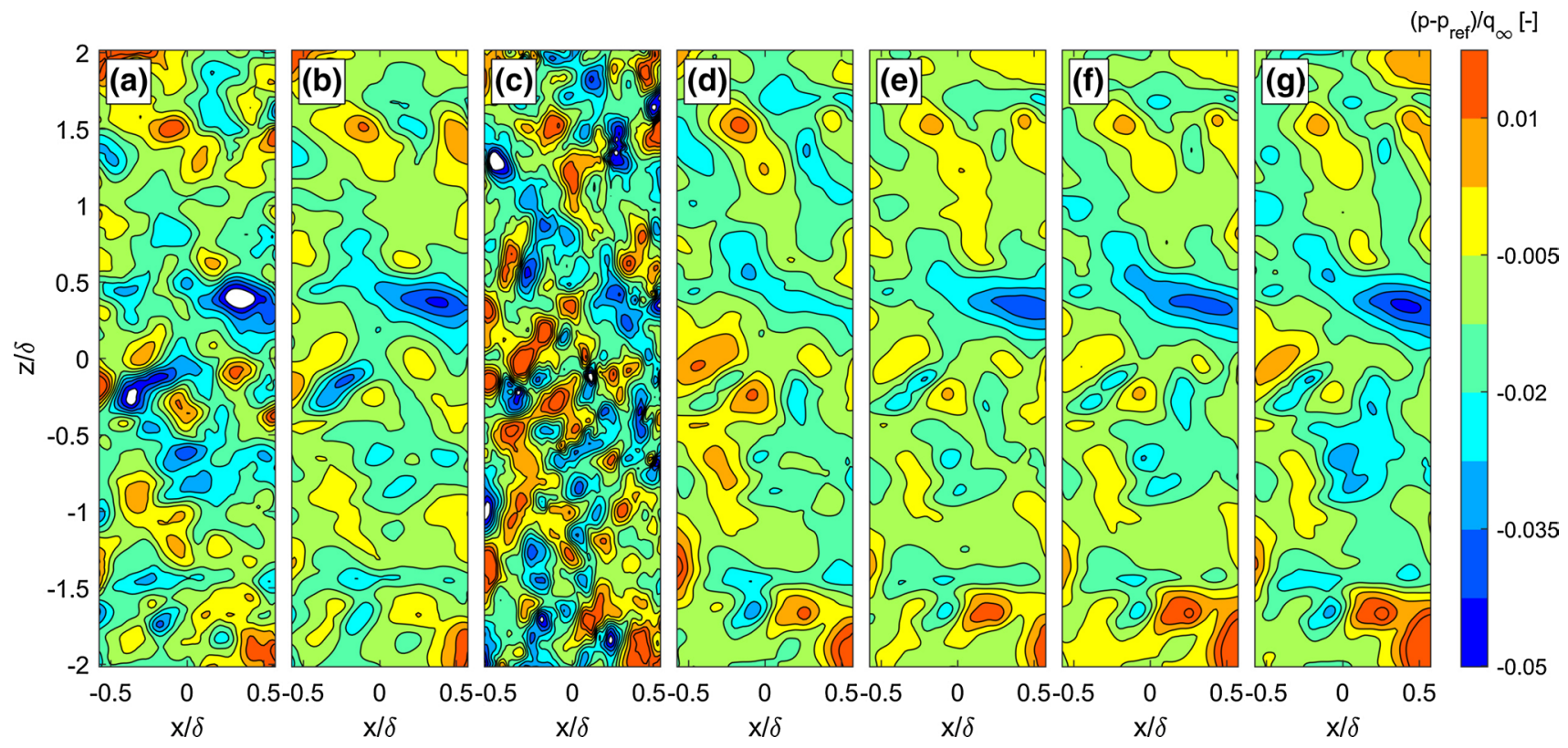

Fig. 7 Comparison of the instantaneous pressure field at $t=2.5 \mathrm{~ms}$ and $y / \delta=0.2$, calculated from, a TR-PIV, $M=1$, b TR-PIV, $M=4$, c PIV, $\mathbf{d}$ PIV + VIC and $\partial \boldsymbol{u} / \partial t=0, \mathbf{e}-\mathbf{g}$ PIV + VIC $(\partial \boldsymbol{u} / \partial t \neq 0)$ with BC type $1-3$

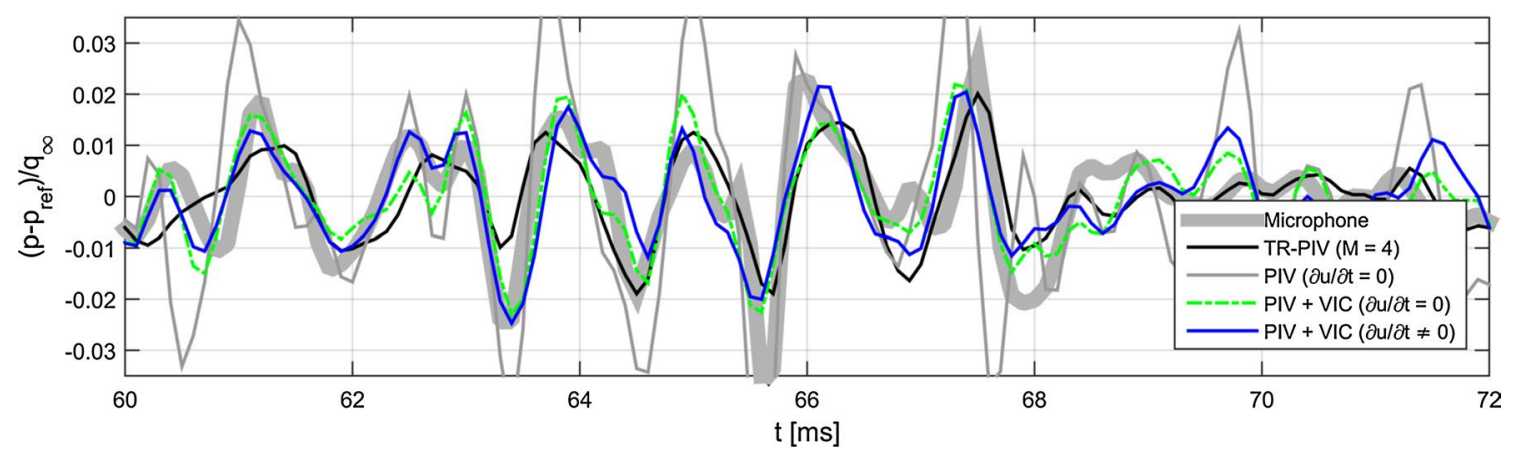

Fig. 8 Pressure fluctuation time-series obtained from TR-PIV, singlesnapshot PIV and single-snapshot PIV + VIC using type 3 boundary conditions, in comparison with the reference microphone measure-

ments at $x / \delta=z / \delta=0$ (grey line, increased thickness for clarity); all results have been band-pass-filtered between 0.3 and $3 \mathrm{kHz}$

Table 3 Correlation coefficient peak values and RMS pressure fluctuation levels

\begin{tabular}{llllll}
\hline Input & Case in Fig. 4 & Stencil size & D $u / \mathrm{D} t$ approximation & $R_{\mathrm{pp}^{\prime}}[-]$ & $\mathrm{p}_{\text {RMS }}^{\prime} / \mathrm{p}_{\text {RMS,ref }}^{\prime}[-]$ \\
\hline TR-PIV & A & 3 snapshots & Lagrangian, $M=1$ & 0.45 & 1.88 \\
TR-PIV & B & 9 snapshots & Lagrangian, $M=4$ & 0.65 & 1.00 \\
PIV & C & 1 snapshot & Eulerian, neglecting $\partial u / \partial t$ & 0.45 & 2.55 \\
PIV & D & 1 snapshot & VIC, $\partial \boldsymbol{u} / \partial t=0$ & 0.62 & 1.31 \\
PIV & E & 1 snapshot & VIC, $\partial \boldsymbol{u} / \partial t \neq 0, \mathrm{BC}=1$ & 0.59 & 0.88 \\
PIV & F & 1 snapshot & VIC, $\partial \boldsymbol{u} / \partial t \neq 0, \mathrm{BC}=2$ & 0.59 & 0.95 \\
PIV & $\mathrm{G}$ & 1 snapshot & VIC, $\partial \boldsymbol{u} / \partial t \neq 0, \mathrm{BC}=3$ & 0.61 & 1.11 \\
\hline
\end{tabular}

is not required for pressure evaluation. However, considering that (1) due to measurement errors velocity divergence is never exactly zero and (2) $\partial \boldsymbol{u} / \partial t$ is needed for Neumann boundary conditions for the Poisson equation for pressure, this is not expected to give acceptable results. To assess this, the approach of entirely neglecting $\partial \boldsymbol{u} / \partial t$, 
$\left.\frac{\mathrm{D} \boldsymbol{u}}{\mathrm{D} t}\right|_{\mathrm{PIV}}=\left(\boldsymbol{u}_{m} \cdot \nabla_{h}\right) \boldsymbol{u}_{m}$,

is also included in the present study. This was attempted before by Imaichi and Ohmi (1983), who reported an increase in error levels and attributed these to neglecting the unsteady term. The present study also finds a low correlation coefficient peak of 0.46 , a significant overestimation of the peak pressure levels (Fig. 7c) in case $\partial \boldsymbol{u} / \partial t$ is neglected, and pressure is calculated directly using the material derivative approximated using (21).

The proposed PIV + VIC single-snapshot procedure is expected to improve upon this. The first part of the procedure regularizes the velocity field $\boldsymbol{u}_{m}$ through Eq. (4) to yield $\boldsymbol{u}_{h}$. The RMS difference between $\boldsymbol{u}_{m}$ and the $\boldsymbol{u}_{h}$ is $0.2 \mathrm{~m} / \mathrm{s}$ at $y / \delta=0.4$, which is considered acceptable for a tomographic PIV experiment at a rather extreme measurement rate of $10 \mathrm{kHz}$. Still neglecting the unsteady term (i.e. setting $\partial \boldsymbol{u} / \partial t=0$ ), this regularized field can be employed to approximate the velocity material derivative,

$\left.\frac{\mathrm{D} \boldsymbol{u}}{\mathrm{D} t}\right|_{\mathrm{VIC} \partial \mathrm{u} / \mathrm{d} t=0}=\left(\boldsymbol{u}_{h} \cdot \nabla_{h}\right) \boldsymbol{u}_{h}$.

Solving pressure with this approximation of the material derivative on the full measurement volume results in a significantly improved correlation coefficient of 0.6 . This approaches the correlation coefficient obtained by the benchmark time-resolved PIV result (Table 3). The instantaneous pressure field depicted in Fig. 7d also shows significant improvement over Fig. 7c. However, the RMS level of the pressure fluctuations, $\mathrm{p}_{\text {RMS }}^{\prime}$, is $31 \%$ larger than the benchmark result.

In the second step of the proposed procedure, $\partial \boldsymbol{u} / \partial t$ is approximated using VIC to allow for approximation of the full velocity material derivative from (7). The velocity material derivative is evaluated on the full domain using the VIC procedure outlined in Sect. 2. Subsequently, as it is expected that the approximation of $\partial \boldsymbol{u} / \partial t$ is less accurate close to the volume boundaries, for pressure evaluation, the volume is cropped by the same amount as for the time-resolved procedure (Sect. 4.1). Three singlesnapshot PIV + VIC cases are discussed, where for cases 1-3, respectively, boundary conditions $1-3$ (Sect. 2.3.2) are employed for the Poisson equation for $\partial \boldsymbol{u} / \partial t$ (Eq. 6) on all volume boundaries except at the wall, where the no-slip condition $(\partial \boldsymbol{u} / \partial t=0)$ is prescribed.

The instantaneous pressure fields approximated using the single-snapshot PIV + VIC procedure with boundary condition types 1-3 are plotted in Fig. 7e-g, respectively. All three approaches yield similar results, indicating a low sensitivity of the VIC procedure to variations in the $\partial \boldsymbol{u} / \partial t$ boundary condition approximation for the present flow case. In addition, minor improvement over the case where the unsteady term was neglected and $\mathbf{u}_{\mathrm{h}}$ was used to solve the steady Poisson equation is visible (e.g. at $x / \delta \approx z / \delta \approx 0.5$ in Fig. 7). The correlation coefficient is, however, not improved further and remains around 0.6, in comparison with 0.65 for the time-resolved benchmark result (Table 3). On the other hand, the inclusion of the approximation of $\partial \boldsymbol{u} / \partial t$ allows for prediction of the RMS pressure fluctuations, with $10 \%$ difference with respect to the time-resolved result, whereas neglecting this term leads to $31 \%$ higher RMS pressure fluctuations (Table 3, last column). For completeness, it should be recalled that in Sect. 2 it was anticipated that when $\partial \omega /\left.\partial t\right|_{h, E u l}$ is employed instead of $\partial \omega /\left.\partial t\right|_{h, V I C}$, a small reduction in quality of the pressure approximation is expected. This has been assessed for the present test case, and only a minor reduction in correlation coefficient was found, i.e. for case 3 boundary conditions, $R_{\mathrm{pp}}$, becomes 0.60 instead of 0.61 .

To assess the correlation of the results in the frequency domain, the magnitude of the coherence of the timeresolved and single-snapshot results is calculated with respect to the microphone signal using Welch's overlapped segment averaging. The result is plotted in Fig. 9 (left figure) and shows that all results have the highest coherence at approximately $0.8-1 \mathrm{kHz}$ and no coherence with the microphone signal for frequencies above $3 \mathrm{kHz}$, where it should be recalled that the results have been band-passfiltered between $300 \mathrm{~Hz}$ and $3 \mathrm{kHz}$. The time-resolved result with $M=4$ shows the expected significant improvement in coherence with respect to $M=1$, with a peak of 0.77 around $900 \mathrm{~Hz}$. Also, the single-snapshot result using VIC improves upon the $M=1$ time-resolved result, showing similar coherence as the time-resolved result using the larger nine-snapshot stencil. The peak coherence around $800 \mathrm{~Hz}$ is approximately $10 \%$ smaller than the benchmark $M=4$ time-resolved result; however, at higher frequency the VIC result follows the trend of the benchmark result. For completeness, the phase lag is also compared in Fig. 9 (right figure), which shows similar results for all procedures.

The higher correlation achieved by the single-snapshot procedure in comparison with the $M=1$ time-resolved procedure is especially relevant for dual-PIV systems (Liu and Katz 2006; Lynch and Scarano 2014), where the limited number of exposures does not allow to regularize the results using a large time-stencil. However, considering that the present study focuses on single-snapshot pressure evaluation, no further speculation is made here that the time-resolved result with $M=1$ may be improved when a larger time separation between the measurements is chosen to reduce the random error component, as discussed in studies by amongst others Jensen and Pedersen (2004) and Perret et al. (2006). 

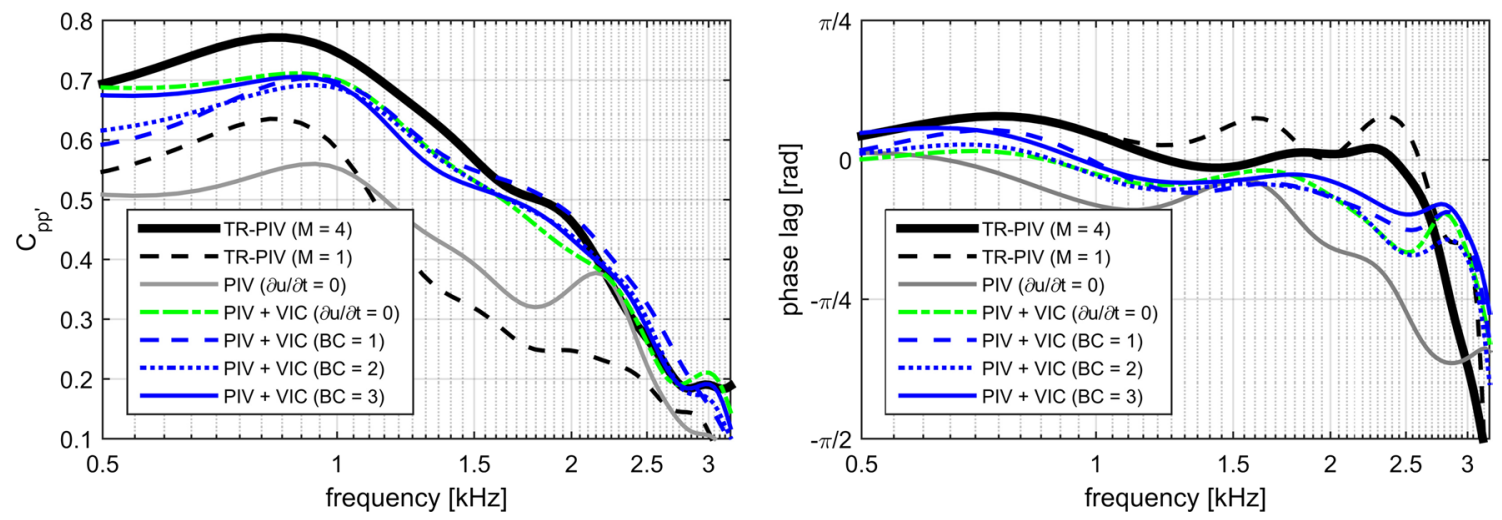

Fig. 9 Coherence (left) and phase of the cross spectrum (right) of the PIV results with the microphone measurements
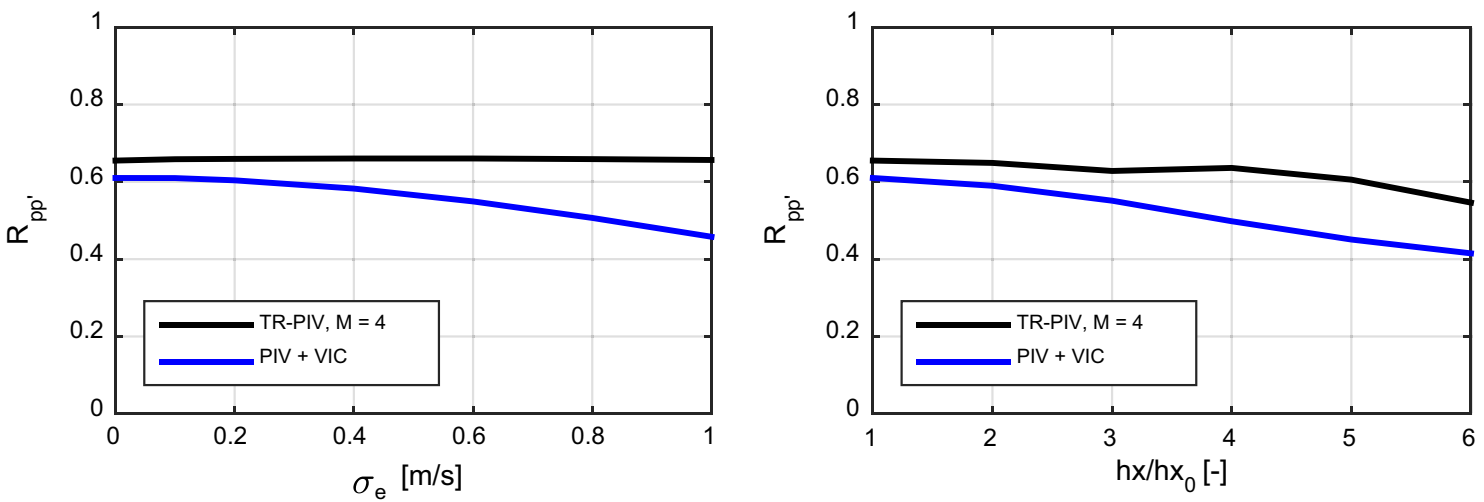

Fig. 10 Measurement sensitivity to measurement noise and spatial resolution. Cross-correlation coefficient with random errors (left) and grid point spacing (right)

To assess the dependence of the method to measurement noise and spatial resolution, here an increase in measurement noise is simulated by adding normally distributed random errors to the velocity vectors and by sub-sampling the velocity fields. Subsequently, the pressure is evaluated as discussed above and the correlation coefficient of the pressure time-series with respect to the microphone measurements is calculated. It is expected that the established time-resolved pressure-from-PIV procedure is less sensitive to these error sources, as multiple PIV velocity snapshots are available for pressure evaluation. Recent literature has shown that the use of multiple snapshots from timeresolved analysis is an effective approach to reduce the effect of measurement errors (e.g. FTEE, Jeon et al. 2014; FTC, Lynch and Scarano 2013).

First, the effect of random noise is considered. Figure 10-left shows the resulting correlation coefficients with respect to the microphone measurements varying the standard deviations of the numerically simulated random errors. The time-resolved procedure is practically insensitive to the random errors, which results from the use of nine consecutive snapshots for pressure evaluation through the least-squares procedure that effectively cancels out the effect of random errors. For the single-snapshot procedure, a gradual reduction of correlation coefficient is observed by increasing the measurement noise. It should be remarked that type 3 boundary conditions have been used for the results plotted. For the other boundary condition types, the same behaviour is observed. A correlation coefficient of approximately 0.5 is retrieved when the error is increased towards $1 \mathrm{~m} / \mathrm{s}$, which corresponds to the order of the turbulent velocity fluctuations close to the microphone location.

The effect of spatial resolution is considered by evaluating the velocity vectors from a coarser grid. Figure 4-right shows the cross-correlation coefficient when the grid point spacing is increased from $\mathrm{hx}_{0}$ in the original data to $\mathrm{hx}$. Both the time-resolved and the single-snapshot methods are sensitive to the spatial resolution. The time-resolved approach still exhibits a plateau up to a fourfold decrease in resolution and a roll-off after that point. Instead, the single-snapshot technique appears to be more sensitive to the decrease in resolution: a small plateau is observed up 
to a twofold decrease in resolution and then a more rapid decrease is observed.

\section{Conclusions}

A method is proposed to approximate the instantaneous flow pressure field from a single instantaneous tomographic PIV velocity snapshot using the vortex-in-cell (VIC) technique and is applied to a flat-plate turbulent boundary layer. By solving the incompressible vorticity transport equation on the measurement volume, the spatial information available from the measurement is leveraged to approximate the temporal velocity derivative. Pressure is subsequently approximated by solution of the Poisson equation for pressure.

The experimental validation compared the correlation coefficient of instantaneous pressure obtained by both the single-snapshot procedure and a benchmark pressure-fromPIV procedure based on time-resolved data, with respect to simultaneous measurement with a surface pressure transducer. The results show that the instantaneous pressure field in a turbulent boundary layer can be evaluated from singlesnapshot PIV, yielding very comparable results to those obtained with time-resolved measurement data acquired at $10 \mathrm{kHz}$ and a stencil of nine consecutive measurements. When a smaller stencil of three consecutive measurements is employed for the time-resolved pressure evaluation, the single-snapshot VIC procedure even outperforms the timeresolved approach, giving a higher cross-correlation with the microphone signal.

The single-snapshot procedure requires a model for the flow acceleration to be used as boundary conditions on the flow governing equations and in the experimental assessment, three such models were assessed: convective, padding and homogeneous boundary conditions, which under the present conditions all yielded similar instantaneous pressure fields. The experimental assessment considered a solid profile along one domain boundary. If the tomographic PIV experiment experiences solid interfaces within the measurement volume, the Poisson solver needs to be adapted accordingly to handle such non-rectangular computational domains.

In the experimental validation, the method yields results corresponding well to those obtained by time-resolved PIV with a nine-snapshot stencil. The advantage of the proposed single-snapshot PIV + VIC procedure is that it does not rely on the acquisition of time-resolved velocity data. Use of the method should be cautioned when the contributions to fluctuating pressure from outside the measurement volume are significant. In the turbulent boundary layer considered here, the proposed method demonstrates that PIV camera and laser hardware requirements can be alleviated, which is relevant for the investigation of high-speed flows where pressure-from-time-resolved-PIV becomes prohibitive.

Acknowledgments The research is partly funded by LaVision $\mathrm{GmbH}$. The work is performed in collaboration with the European FP-7 project "NIOPLEX", Grant Agreement 605,151.

Open Access This article is distributed under the terms of the Creative Commons Attribution 4.0 International License (http://creativecommons.org/licenses/by/4.0/), which permits unrestricted use, distribution, and reproduction in any medium, provided you give appropriate credit to the original author(s) and the source, provide a link to the Creative Commons license, and indicate if changes were made.

\section{References}

Atkinson C, Coudert S, Foucaut JM, Stanislas M, Soria J (2011) The accuracy of tomographic particle image velocimetry for measurements of a turbulent boundary layer. Exp Fluids 50:1031-1056

Bai Z, Wimalajeewa T, Berger Z, Wang G, Glauser M, Varshney PK (2015) Low-dimensional approach for reconstruction of airfoil data via compressive sensing. AIAA J 53:4

Charonko JJ, King CV, Smith BL, Vlachos PP (2010) Assessment of pressure field calculations from particle image velocimetry measurements. Meas Sci Technol 2010:105401

Cottet G-H, Poncet P (2003) Advances in direct numerical simulations of 3D wall-bounded flows by Vortex-in-Cell methods. J Comput Phys 193:136-158

de Kat R, Ganapathisubramani B (2013) Pressure from particle image velocimetry for convective flows: a Taylor's hypothesis approach. Meas Sci Technol 24:024002

de Kat R, van Oudheusden BW (2012) Instantaneous planar pressure determination from PIV in turbulent flow. Exp Fluids 52:1089-1106

de Silva CM, Philip J, Marusic I (2013) Minimization of divergence error in volumetric velocity measurements and implications for turbulence statistics. Exp Fluids 54:1557

Ebbers T, Farnebäck G (2009) Improving computation of cardiovascular relative pressure fields from velocity MRI. J Magn Reson Imaging 30:54-61

Ghaemi S, Scarano F (2010) Multi-pass light amplification for tomographic particle image velocimetry applications. Meas Sci Technol 21:127002

Ghaemi S, Scarano F (2011) Counter-hairpin vortices in the turbulent wake of a sharp trailing edge. J Fluid Mech 689:317-356

Ghaemi and Scarano (2013) Turbulent structure of high-amplitude pressure peaks within the turbulent boundary layer. J Fluid Mech 735:381-426

Ghaemi S, Ragni D, Scarano F (2012) PIV-based pressure fluctuations in the turbulent boundary layer. Exp Fluids 53:1823-1840

Gronskis A, Heitz D, Mémin E (2013) Inflow and initial conditions for direct numerical simulation based on adjoint data assimilation. J Comput Phys 242:480-497

Imaichi K, Ohmi K (1983) Numerical processing of flow-visualization pictures-measurement of two-dimensional vortex flow. J Fluid Mech 129:283-311

Jensen A, Pedersen GK (2004) Optimization of acceleration measurements using PIV. Meas Sci Technol 15:2275-2283

Kähler CJ, Kompenhans J (2000) Fundamentals of multiple plane stereo particle image velocimetry. Exp Fluids 29:S70-S77 
Krogstad P-A, Kaspersen JH, Rimestad S (1998) Convection velocities in a turbulent boundary layer. Phys Fluids 10:949-957

Kurtulus DF, Scarano F, David L (2007) Unsteady aerodynamic forces estimation on a square cylinder by TR-PIV. Exp Fluids 42:185-196

Lemke M, Sesterhenn J (2013) Adjoint based pressure determination from PIV-data - validation with synthetic PIV measurements. In: 10th international symposium on PIV, Delft, The Netherlands

Liu X, Katz J (2006) Instantaneous pressure and material acceleration measurements using a four-exposure PIV system. Exp Fluids 41:227-240

Liu X, Katz J (2013) Vortex-corner interactions in a cavity shear layer elucidated by time-resolved measurements of the pressure field. J Fluid Mech 728:417-457

Lynch K, Scarano F (2014) Material acceleration estimation by fourpulse tomo-PIV. Meas Sci Technol 25:084005

Marusic I, McKeon BJ, Monkewitz PA, Nagib HM, Smits AJ, Sreenivasan KR (2010) Wall-bounded turbulent flows at high reynolds numbers: recent advances and key issues. Phys Fluids 22:065103

Novara M, Scarano F (2013) A particle-tracking approach for accurate material derivative measurements with tomographic PIV. Exp Fluids 54:1584

Orlanski I (1976) A simple boundary condition for unbounded hyperbolic flows. J Comput Phys 21:251-269

Perret L, Braud P, Fourment C, David L, Delville J (2006) 3-Component acceleration field measurement by dual-time stereoscopic particle image velocimetry. Exp Fluids 40:813-824

Pröbsting S, Scarano F, Bernardini M, Pirozzoli S (2013) On the estimation of wall pressure coherence using time-resolved tomographic PIV. Exp Fluids 54:1567

Ragni D, Ashok A, van Oudheusden BW, Scarano F (2009) Surface pressure and aerodynamic loads determination of a transonic airfoil based on particle image velocimetry. Meas Sci Technol 20:074005
Scarano F (2013) Tomographic PIV: principles and practice. Meas Sci Technol 24:012001

Scarano F, Moore P (2011) An advection-based model to increase the temporal resolution of PIV time series. Exp Fluids 52:919-933

Schiavazzi D, Coletti F, Iaccarino G, Eaton JK (2014) A matching pursuit approach to solenoidal filtering of three-dimensional velocity measurements. J Comput Phys 263:206-221

Schneiders JFG, Dwight RP, Scarano F (2014) Time-supersampling of 3D-PIV measurements with vortex-in-cell simulation. Exp Fluids 55:1692

Schröder A, Geisler R, Elsinga GE, Scarano F, Dierksheide U (2008) Investigation of a turbulent spot and a tripped turbulent boundary layer flow using time-resolved tomographic PIV. Exp Fluids 44:305-316

Sciacchitano A, Lynch KP (2015) A posteriori uncertainty quantification for tomographic-PIV data. In: 11th international symposium particle image velocimetry, Santa Barbara, California, Sept 14-16

Sciacchitano A, Dwight RP, Scarano F (2012) Navier-Stokes simulations in gappy PIV data. Exp Fluids 53:1421-1435

Suzuki T (2012) Reduced-order Kalman-filtered hybrid simulation combining particle tracking velocimetry and direct numerical simulation. J Fluid Mech 709:249-288

Suzuki T (2014) POD-based reduced-order hybrid simulation using the data- driven transfer function with time-resolved PTV feedback. Exp Fluids 55:1798

van Oudheusden BW (2013) PIV-based pressure measurement. Meas Sci Technol 24:032001

Violato D, Moore P, Scarano F (2011) Lagrangian and Eulerian pressure field evaluation of rod-airfoil flow from time-resolved tomographic PIV. Exp Fluids 50:1057-1070

Vlasenko A, Steele ECC, Nimmo-Smith WAM (2015) A physics-enabled flow restoration algorithm for sparse PIV and PTV measurements. Meas Sci Technol 2015:065301

Wills JAB (1964) On convection velocities in turbulent shear flows. J Fluid Mech 20:417-432 